Simon

Preismanagement 


\title{
Hermann Simon
}

\section{Preismanagement}

\author{
Analyse - Strategie - Umsetzung
}

2., vollständig überarbeitete und erweiterte Auflage 
Prof. Dr. Hermann Simon ist Inhaber des Lehrstuhls für Betriebswirtschaftslehre und Marketing an der Johannes Gutenberg-Universität Mainz.

Die Deutsche Bibliothek - CIP-Einheitsaufnahme

\author{
Simon, Hermann: \\ Preismanagement: Analyse, Strategie, \\ Umsetzung/Hermann Simon. - \\ 2., vollst. überarb. und erw. Aufl. - Wiesbaden: \\ Gabler, 1992 \\ ISBN 978-3-322-99740-1 ISBN 978-3-322-99739-5 (eBook) \\ DOI 10.1007/978-3-322-99739-5
}

1. Auflage 1982

2. Auflage 1992

Der Gabler Verlag ist ein Unternehmen der Verlagsgruppe Bertelsmann International.

(C) Hermann Simon 1992

Softcover reprint of the hardcover 2nd edition 1992

Lektorat: Gudrun Böhler

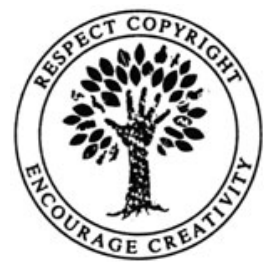

Das Werk einschließlich aller seiner Teile ist urheberrechtlich geschützt. Jede Verwertung außerhalb der engen Grenzen des Urheberrechtsgesetzes ist ohne Zustimmung des Verlages unzulässig und strafbar. Das gilt insbesondere für Vervielfältigungen, Übersetzungen, Mikroverfilmungen und die Einspeicherung und Verarbeitung in elektronischen Systemen.

Höchste inhaltliche und technische Qualität unserer Produkte ist unser Ziel. Bei der Produktion und Verbreitung unserer Bücher wollen wir die Umwelt schonen: Dieses Buch ist auf säurefreiem und chlorfrei gebleichtem Papier gedruckt. Die Einschweißfolie besteht aus Polyäthylen und damit aus organischen Grundstoffen, die weder bei der Herstellung noch bei der Verbrennung Schadstoffe freisetzen.

Die Wiedergabe von Verbrauchsnamen, Handelsnamen, Warenbezeichnungen usw. in diesem Werk berechtigt auch ohne besondere Kennzeichnung nicht zu der Annahme, daß solche Namen im Sinne der Warenzeichen- und Markenschutz-Gesetzgebung als frei zu betrachten wären und daher von jedermann benutzt werden dürften. 


\section{Vorwort}

Die Bedeutung des Preises hat in vielen modernen Märkten zugenommen. Dieser Trend hält an. Ursächlich hierfür sind reale Entwicklungen wie Marktsättigung, Qualitätsangleichung und Globalisierung. Unternehmen werden immer stärker gezwungen, den Preis als Wettbewerbswaffe einzusetzen. Befragungen zeigen, daß der von Managern beim Marketinginstrument Preis empfundene Druck ständig anwächst.

In den zehn Jahren seit Erscheinen der ersten Auflage dieses Buches hat es in der Preistheorie und insbesondere in der praktischen Anwendung neuer Konzepte enorme Fortschritte gegeben. Die traditionell große Lücke zwischen einer hochentwickelten Preistheorie und einer unterentwickelten Preisbildungspraxis ist zumindest in fortschrittlichen Unternehmen kleiner geworden. Wir besitzen heute nicht nur weitaus bessere theoretische Methoden wie Conjoint Measurement oder nichtlineare Preisbildung, sondern konnten umfangreiche Erfahrungen in der Anwendung dieser Methoden gewinnen.

Im vorliegenden Buch habe ich mir als Ziel gesetzt, sowohl den neuesten State of the Art des Preismanagements darzustellen als auch die Problematik der praktischen Umsetzung $\mathrm{zu}$ behandeln. Gegenüber der ersten Auflage haben sich folglich die Schwerpunkte verschoben, und es sind neue Themenkreise aufgenommen worden. Die wichtigsten Entwicklungen der letzten zehn Jahre bestehen darin, einerseits durch eine fundierte Nutzenmessung Preisbereitschaften abzuschätzen und andererseits Preise so zu differenzieren, daß Gewinnpotentiale möglichst vollständig ausgeschöpft werden. Neben einer Einordnung der Preispolitik in die übergeordnete Wettbewerbsstrategie nimmt demgemäß die Darstellung von Methoden zur preisorientierten Nutzenmessung breiten Raum ein. Der Bereich Preisdifferenzierung wurde von einem auf vier Kapitel erweitert. Neben der Marktsegmentierung werden internationale Preisbildungsprobleme, die Preispolitik für Produktlinien sowie die nichtlineare Preisbildung behandelt. Intensiv wird auf branchenspezifische Preisbildungsprobleme eingegangen. Den Branchen Industriegüter, Dienstleistungen und Handel sind jeweils eigene Kapitel gewidmet. Ein separates Kapitel beschäftigt sich mit der Umsetzung, die mit höheren methodischen Ansprüchen komplizierter wird.

Das vorliegende Buch wäre nicht möglich gewesen ohne die jahrelange Zusammenarbeit mit der UNIC-University Connection Institut für Management und Marketing GmbH in Bonn. Die Mitarbeiter von UNIC haben in den unterschiedlichsten Branchen Preisentscheidungen, die aggregierte Umsätze von mehreren hundert Milliarden DM betreffen, mitgestaltet. Das UNIC-Team dürfte die weltweit umfangreichste Erfahrung in der Anwendung hochentwickelter Methoden zur Unterstützung des Preismanagements besitzen. Viele der gewonnenen Erfahrungen sind in das vorliegende Buch eingeflossen. Ich danke den Herren Dr. Eckhard Kucher, Dr. Karl-Heinz Sebastian, Dr. Klaus Hilleke- 
Daniel, Dr. Georg Tacke, Dr. Michael Laker und Dr. Bernhard Ebel, daß sie mich an diesen Erfahrungen partizipieren ließen. Mein Dank gilt auch Professor Robert Dolan für wertvolle Anregungen und Gedanken, insbesondere während meiner Gastprofessur an der Harvard Business School. Professor Pil Yoo von der Sung Kyun Kwan Universität in Seoul und Professor Gert Assmus vom Dartmouth College verdanke ich wichtige Verbesserungsvorschläge.

Die völlige Neubearbeitung des Buches wäre ohne die Unterstützung meiner Mitarbeiter am Lehrstuhl nicht möglich gewesen. Zu besonderem Dank bin ich Diplom-Kaufmann Carsten Wiese und Diplom-Wirtschaftsingenieur Martin Möhrle verpflichtet. Genauso war die Mitarbeit von Diplom-Kaufmann Martin Faßnacht, cand. rer. pol. Veronika Speckmann, cand. rer. pol. Stefan Böttcher und meiner Sekretärin Corinna Axt unverzichtbar. Ihnen allen danke ich herzlich.

Nicht zuletzt geht ein Dankeschön an Cäcilia, Jeannine und Patrick, die ihren Beitrag zu diesem Buch in Form von entbehrter Zeit und großem Verständnis leisteten.

HERMANN SIMON 


\section{Inhaltsverzeichnis}

\section{Erster Teil}

Einführung in das Preismanagement $\ldots \ldots \ldots \ldots \ldots \ldots \ldots \ldots \ldots \ldots \ldots \ldots \ldots \ldots$

Erstes Kapitel

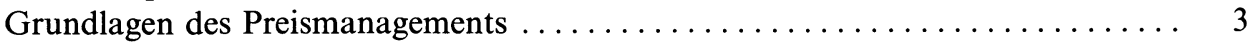

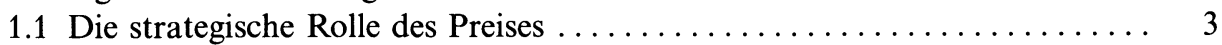

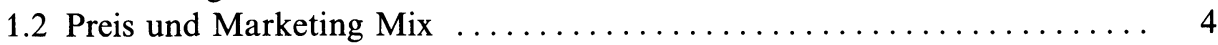

1.3 Zunehmende Bedeutung des Preises $\ldots \ldots \ldots \ldots \ldots \ldots \ldots \ldots \ldots \ldots \ldots$

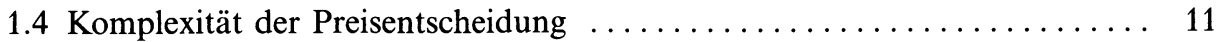

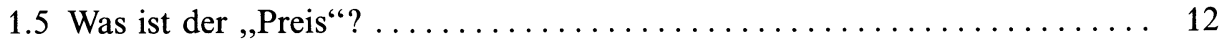

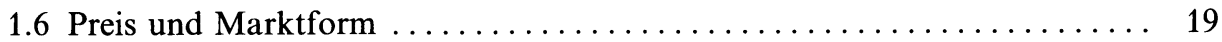

1.7 Preismanagement in den Wirtschaftswissenschaften $\ldots \ldots \ldots \ldots \ldots \ldots 23$

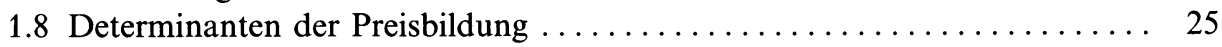

Zweites Kapitel

Preismanagement im Überblick $\ldots \ldots \ldots \ldots \ldots \ldots \ldots \ldots \ldots \ldots \ldots \ldots \ldots \ldots . \ldots \ldots$

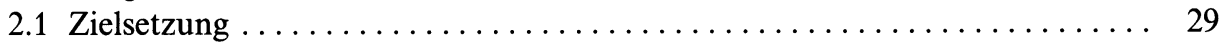

2.2 Strukturierung des Preisbildungsproblems $\ldots \ldots \ldots \ldots \ldots \ldots \ldots \ldots \ldots . \ldots \ldots$

2.3 Preismanagement unter statischen Bedingungen $\ldots \ldots \ldots \ldots \ldots \ldots \ldots \ldots$

2.3.1 Die statische Preisabsatzfunktion $\ldots \ldots \ldots \ldots \ldots \ldots \ldots \ldots \ldots \ldots \ldots$

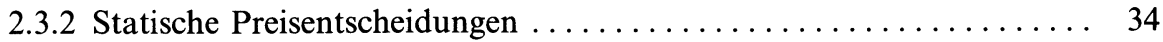

2.4 Preismanagement unter dynamischen Bedingungen $\ldots \ldots \ldots \ldots \ldots \ldots \ldots$

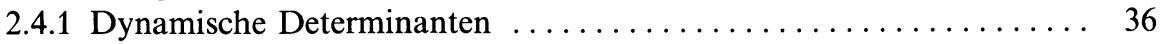

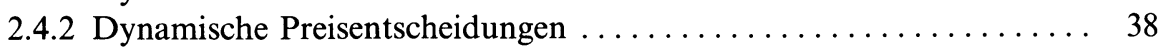

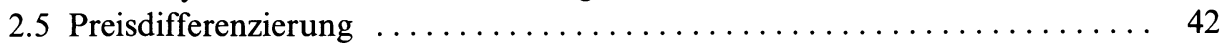

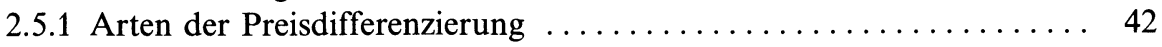

2.5.2 Preisdifferenzierung nach Marktsegmenten $\ldots \ldots \ldots \ldots \ldots \ldots \ldots . \ldots 2$

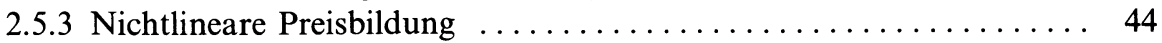

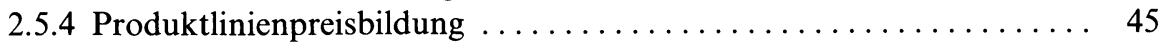

2.5.5 Internationale Preisdifferenzierung $\ldots \ldots \ldots \ldots \ldots \ldots \ldots \ldots \ldots$

2.6 Branchenspezifische Preisbildungsprobleme $\ldots \ldots \ldots \ldots \ldots \ldots \ldots \ldots \ldots . \ldots \ldots$

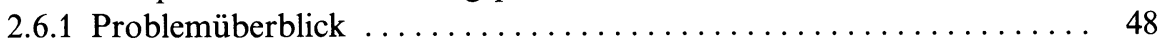

2.6.2 Preismanagement und Handel $\ldots \ldots \ldots \ldots \ldots \ldots \ldots \ldots \ldots \ldots \ldots$

2.6.3 Preismanagement für Industriegüter $\ldots \ldots \ldots \ldots \ldots \ldots \ldots \ldots \ldots \ldots$

2.6.4 Preismanagement für Dienstleistungen $\ldots \ldots \ldots \ldots \ldots \ldots \ldots \ldots \ldots$

2.7 Spezial- und Umsetzungsprobleme $\ldots \ldots \ldots \ldots \ldots \ldots \ldots \ldots \ldots \ldots \ldots \ldots$

2.7.1 Preismanagement und Psychologie $\ldots \ldots \ldots \ldots \ldots \ldots \ldots \ldots \ldots \ldots$

2.7.2 Preismanagement und Marketing Mix-Interaktion .......... 55

2.7.3 Umsetzungsprobleme ..................... 56 
Drittes Kapitel

Preismanagement und Strategie $\ldots \ldots \ldots \ldots \ldots \ldots \ldots \ldots \ldots \ldots \ldots \ldots \ldots$

3.1 Der Preis als Element der Wettbewerbsstrategie $\ldots \ldots \ldots \ldots \ldots \ldots \ldots \ldots$. . . . . 59

3.2 Preis und Wettbewerbsvorteil .......................... 66

3.3 Preisinformation und Wettbewerbsstrategie $\ldots \ldots \ldots \ldots \ldots \ldots \ldots \ldots \ldots$

3.4 Langfristige Preisentwicklungen $\ldots \ldots \ldots \ldots \ldots \ldots \ldots \ldots \ldots \ldots \ldots$

\section{Zweiter Teil}

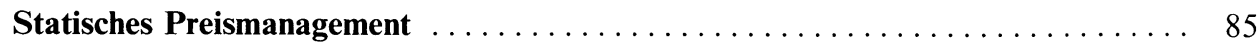

Viertes Kapitel

Die statische Preisabsatzfunktion $\ldots \ldots \ldots \ldots \ldots \ldots \ldots \ldots \ldots \ldots \ldots \ldots$

4.1 Systemzusammenhang der statischen Preisentscheidung . . . . . . . . 87

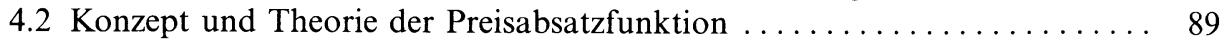

4.2.1 Klassifikation von Preisabsatzfunktionen . . . . . . . . . . . . 89

4.2.2 Theoretische Begründung der Preisabsatzfunktion . . . . . . . . . . 90

4.2.3 Mathematische Preisabsatzfunktionen ..................... 94

4.3 Empirische Bestimmung der Preisabsatzfunktion ............... 109

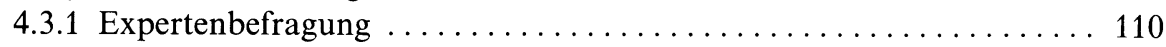

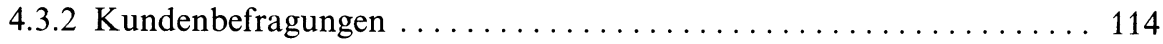

4.3.3 Preisexperimente ............................ 126

4.3 .4 Marktdaten ................................ 131

4.4 Empirische Befunde zur Preiselastizität ................... 137

Fünftes Kapitel

Statisches Preismanagement unter monopolistischen Bedingungen ......... 143

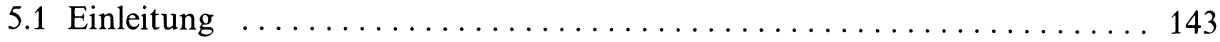

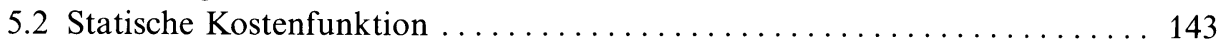

5.3 Statische Preisuntergrenzen . . . . . . . . . . . . . . . . . . . . 147

5.4 Statisches Preismanagement mit Hilfsverfahren . . . . . . . . . . . . . . . 149

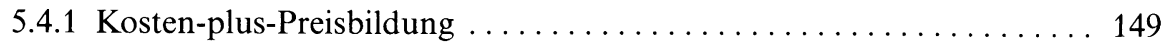

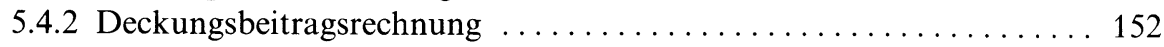

5.4 .3 Entscheidungsbaumverfahren ................... 158

5.4 .4 Maximalpreisorientierte Preisbildung . . . . . . . . . . . . 160

5.5 Statisches Preismanagement mit exakten Verfahren $\ldots \ldots \ldots \ldots \ldots \ldots \ldots 2$

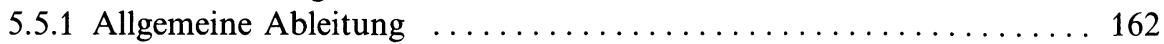

5.5.2 Lineare Preisabsatzfunktion . ..................... 164

5.5.3 Multiplikative Preisabsatzfunktion $\ldots \ldots \ldots \ldots \ldots \ldots \ldots \ldots \ldots \ldots$

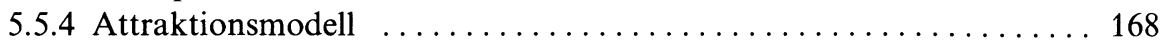

5.5 .5 Gutenberg-Modell ............................ 169

5.6 Spezialprobleme des statischen Preismanagements $\ldots \ldots \ldots \ldots \ldots \ldots \ldots 176$

5.6.1 Die Steuerung der Kapazitätsauslastung mit Hilfe des Preises ... . . 177

5.6.2 Preismanagement und Steuern .................... 178

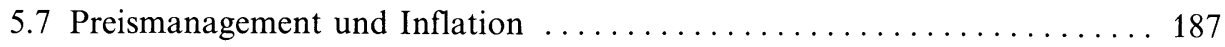

5.7.1 Kalkulation zu Anschaffungs- oder zu Wiederbeschaffungskosten .. 188 
5.7.2 Kosteninflation und Preisabsatzfunktion ............... 191

5.7.3 Inflation und Preistaktik . . . . . . . . . . . . . . . . . . 194

Sechstes Kapitel

Statisches Preismanagement unter oligopolistischen Bedingungen . . . . . . . . . 199

6.1 Systemzusammenhang . . . . . . . . . . . . . . . . . . . . 199

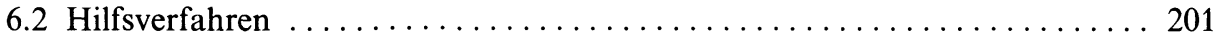

6.3 Oligopolistisches Preismanagement mit exakten Verfahren .......... 205

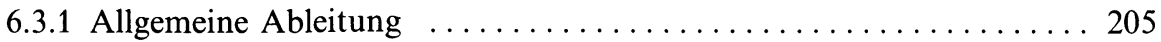

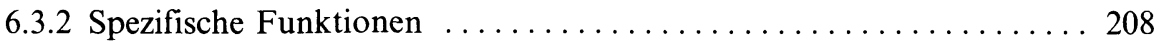

6.4 Oligopoltheoretische Reaktionshypothesen $\ldots \ldots \ldots \ldots \ldots \ldots \ldots \ldots . \ldots 214$

6.4.1 Oligopolistisches Preismanagement bei linearen

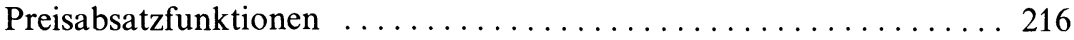

6.4.2 Oligopolistisches Preismanagement bei

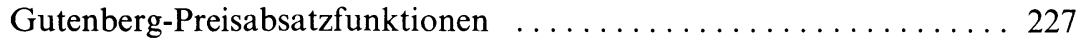

\section{Dritter Teil}

Dynamisches Preismanagement

Siebtes Kapitel

Determinanten des dynamischen Preismanagements ................. 239

7.1 Probleme und Besonderheiten der dynamischen Betrachtung . . . . . . . . 239

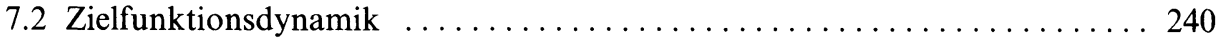

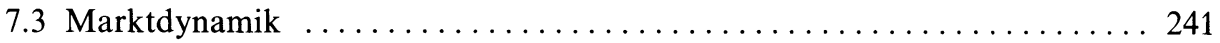

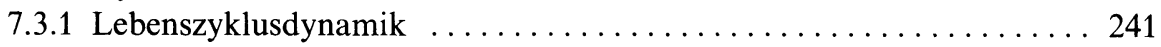

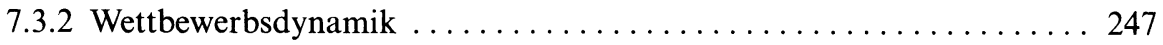

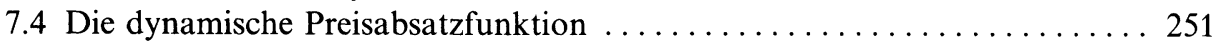

7.4.1 Dynamische Dimensionen der Preiswirkung $\ldots \ldots \ldots \ldots \ldots \ldots \ldots 251$

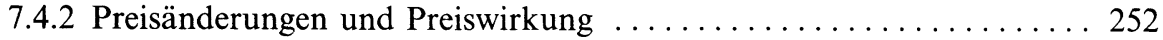

7.4 .3 Carryover-Effekte ............................ 258

7.4.4 Preiswirkung und Lebenszyklus ................... 264

7.5 Zur Dynamik der Preiselastizitäten . . . . . . . . . . . . . . 275

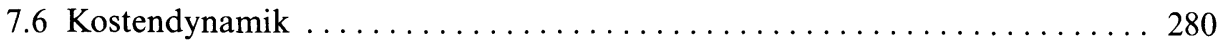

Achtes Kapitel

Dynamisches Preismanagement . . . . . . . . . . . . . . . . . . . . 291

8.1 Systemzusammenhang . . . . . . . . . . . . . . . . . . . . . 291

8.2 Dynamisches Preismanagement mit Hilfsverfahren ............... 293

8.3 Fundamentale Optimalitätsregeln für das dynamische Preismanagement . . 300

8.3.1 Dynamisch-optimaler Preis bei allgemeiner dynamischer

Preisabsatzfunktion ........................ 300

8.3.2 Dynamisch-optimaler Preis bei Carryover-Effekten . . . . . . . . . . 304

8.3.3 Dynamisch-optimaler Preis bei Preisänderungswirkungen ........ . 309

8.3.4 Dynamisch-optimaler Preis bei Kostendynamik ............. 313

8.3.5 Zur Existenz dynamischer Preisuntergrenzen .............. 316

8.3.6 Zusammenfassung der dynamischen Fundamentalregeln . . . . . . . 318 
8.4 Dynamisches Preismanagement und Lebenszyklus . . . . . . . . . . . 320

8.4.1 Dynamisches Preismanagement bei echten Innovationen . . . . . . . 320

8.4.2 Dynamisches Preismanagement und Konkurrenz . . . . . . . . . . 331

8.4.3 Dynamisches Preismanagement in bestehenden Märkten ....... 346

\section{Vierter Teil}

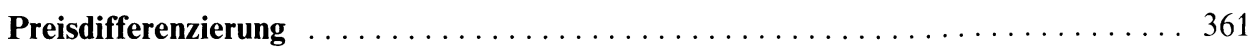

Neuntes Kapitel

Preismanagement und Marktsegmentierung $\ldots \ldots \ldots \ldots \ldots \ldots \ldots \ldots \ldots \ldots$

9.1 Problemstellung der Marktsegmentierung $\ldots \ldots \ldots \ldots \ldots \ldots \ldots \ldots \ldots \ldots$

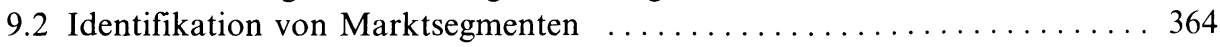

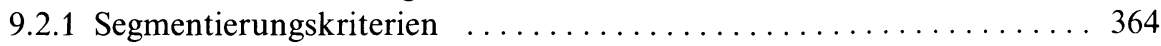

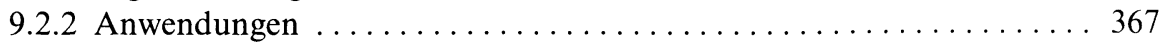

9.3 Preisdifferenzierung nach Marktsegmenten $\ldots \ldots \ldots \ldots \ldots \ldots \ldots \ldots . \ldots \ldots 1$

9.3.1 Normative Aspekte der Preisdifferenzierung . . . . . . . . . . . . 382

9.3.2 Operationale Aspekte der Preisdifferenzierung . . . . . . . . . . . . 391

9.3.3 Juristische Aspekte der Preisdifferenzierung . . . . . . . . . . . . . 396

Zehntes Kapitel

Nichtlineare Preisbildung . . . . . . . . . . . . . . . . . . . . . . 399

10.1 Zielsetzung und Formen der nichtlinearen Preisbildung . . . . . . . . . 399

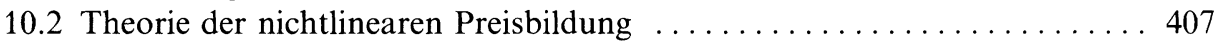

10.3 Umsetzung der nichtlinearen Preisbildung $\ldots \ldots \ldots \ldots \ldots \ldots \ldots \ldots \ldots \ldots$

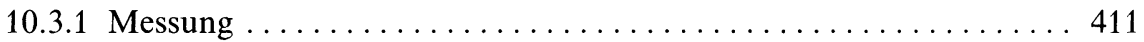

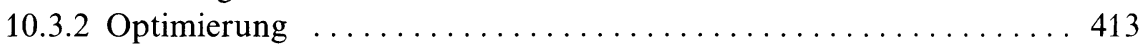

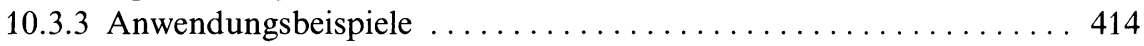

10.3.4 Umsetzungsprobleme . . . . . . . . . . . . . . . . . . . . 419

Elftes Kapitel

Preismanagement für Produktlinien $\ldots \ldots \ldots \ldots \ldots \ldots \ldots \ldots \ldots \ldots \ldots \ldots \ldots \ldots$

11.1 Preisbildungsprobleme im Mehrproduktunternehmen ........... 423

11.2 Optimierung der Preise in einer Produktlinie $\ldots \ldots \ldots \ldots \ldots \ldots \ldots \ldots 26$

11.2.1 Das Optimierungsproblem .................... 426

11.2.2 Beispiele für produktübergreifende Preisoptimierung . . . . . . . . 429

11.2.3 Vereinfachung bei Mengenverbund ................ 438

11.2.4 Umsetzungsprobleme bei produktübergreifender Preisoptimierung 440

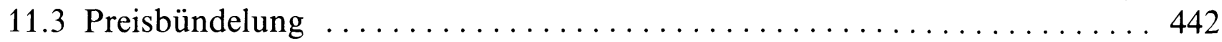

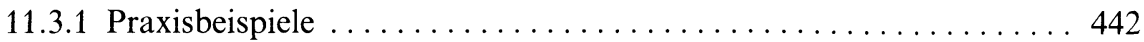

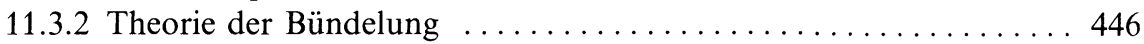

11.3.3 Anwendungs- und Umsetzungsprobleme der Produkt- und

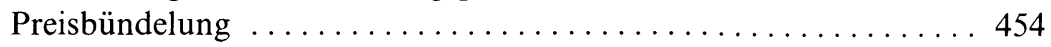

Zwölftes Kapitel

Internationales Preismanagement $\ldots \ldots \ldots \ldots \ldots \ldots \ldots \ldots \ldots \ldots \ldots \ldots \ldots$

12.1 Preismanagement und internationales Geschäft . . . . . . . . . . . . . 459 
12.2 Inflation und internationales Preismanagement $\ldots \ldots \ldots \ldots \ldots \ldots \ldots 6$

12.3 Wechselkurs und internationales Preismanagement . . . . . . . . . 468

12.3.1 Wechselkurs und optimaler Preis . . . . . . . . . . . . 468

12.3.2 Folgen nichtoptimaler Preispolitik bei Wechselkursänderungen . . 471

12.3.3 Empirische Beispiele zum Zusammenhang von Preis

und Wechselkurs ........................... 474

12.4 Parallelimporte und internationales Preismanagement . . . . . . . . . . 476

12.5 Staatliche Eingriffe und internationales Preismanagement . . . . . . . . 481

12.6 Umsetzungsaspekte im internationalen Preismanagement . . . . . . . . 486

\section{Fünfter Teil}

Branchenspezifisches Preismanagement $\ldots \ldots \ldots \ldots \ldots \ldots \ldots \ldots \ldots \ldots$

Dreizehntes Kapitel

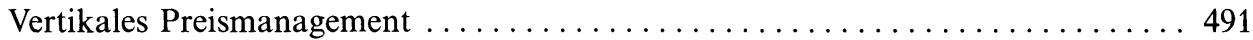

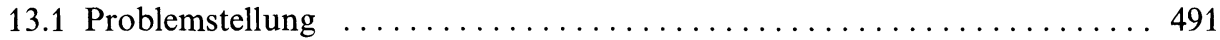

13.2 Vertikales Preismanagement des Herstellers . . . . . . . . . . . . . . 493

13.2.1 Der Hersteller bestimmt Herstellerabgabe- und Endpreis . . . . . . 493

13.2.2 Der Hersteller bestimmt nur den Herstellerabgabepreis . . . . . . . 501

13.2.3 Hersteller und Handel betreiben gemeinsame Gewinnmaximierung 508

Vierzehntes Kapitel

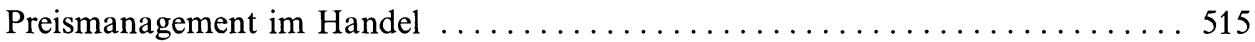

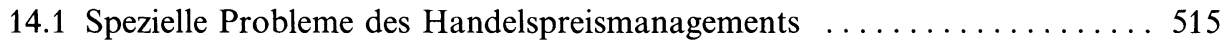

14.2 Artikelbezogenes Preismanagement im Handel ............... 516

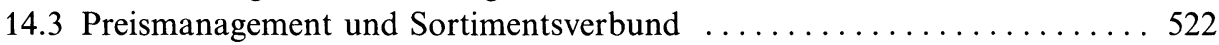

14.4 Sonderangebote des Handels $\ldots \ldots \ldots \ldots \ldots \ldots \ldots \ldots \ldots \ldots \ldots \ldots \ldots 26$

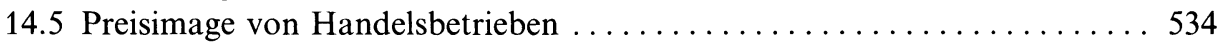

Fünfzehntes Kapitel

Preismanagement für Industriegüter $\ldots \ldots \ldots \ldots \ldots \ldots \ldots \ldots \ldots \ldots \ldots \ldots \ldots \ldots \ldots \ldots \ldots \ldots$

15.1 Besonderheiten des Preismanagements für Industriegüter $\ldots \ldots \ldots \ldots \ldots 41$

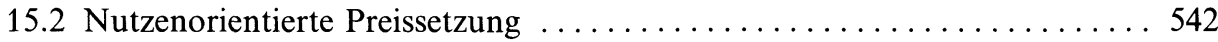

15.2.1 Preis-Leistungs-Verhältnisse als Preisbildungsbasis . . . . . . . . 543

15.2.2 Wirtschaftlichkeitsrechnung als Preisbildungsbasis ........... 549

15.3 Preisaushandlung, Preistransparenz und oligopolistische Reaktion ..... 553

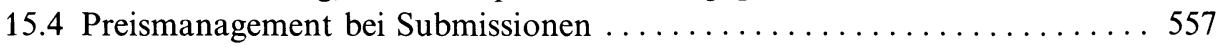

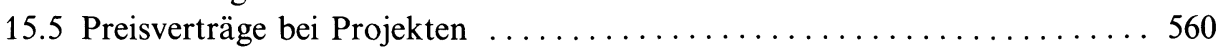

Sechzehntes Kapitel

Preismanagement für Dienstleistungen $\ldots \ldots \ldots \ldots \ldots \ldots \ldots \ldots \ldots \ldots \ldots \ldots$

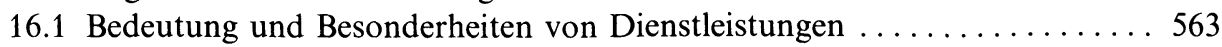

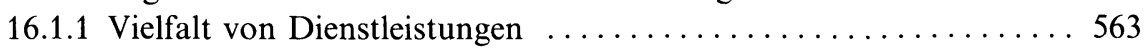

16.1.2 Preispolitische Besonderheiten von Dienstleistungen $\ldots \ldots \ldots \ldots 565$ 


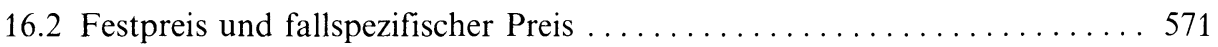

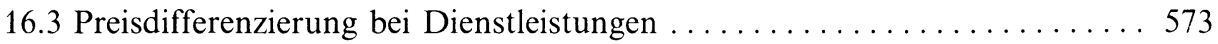

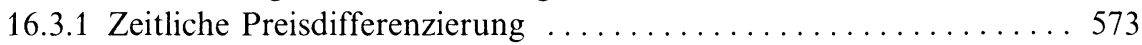

16.3.2 Spitzenlast-Preisbildung ....................... 574

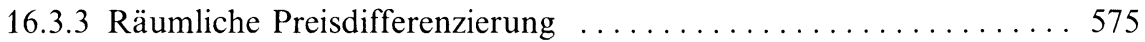

16.3.4 Personenbezogene Preisdifferenzierung .............. 576

16.3.5 Leistungsbezogene Preisdifferenzierung . . . . . . . . . . . . 576

16.3.6 Mengenbezogene Preisdifferenzierung . . . . . . . . . . . . . 577

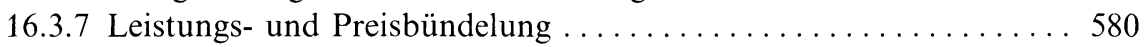

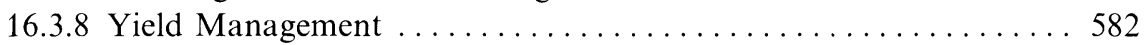

16.4 Dynamisches Preismanagement bei Dienstleistungen $\ldots \ldots \ldots \ldots \ldots \ldots 58$

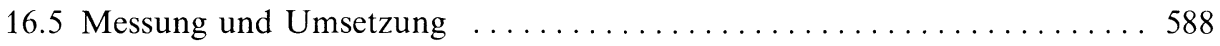

\section{Sechster Teil}

Spezialprobleme des Preismanagements . . . . . . . . . . . . . . . . . . . . . . 589

Siebzehntes Kapitel

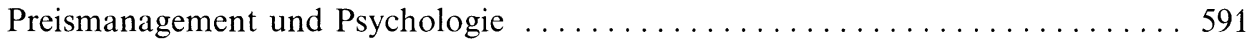

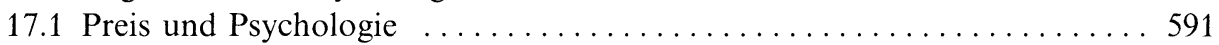

17.2 Preiswahrnehmung und Preisbeurteilung ................. 591

17.2.1 Das System der Preiswahrnehmung und -beurteilung . . . . . . . . 592

17.2.2 Einflußgrößen von Preiswahrnehmung und -beurteilung . . . . . . 594

17.2.3 Steuerung von Preiswahrnehmung und -beurteilung .......... 599

17.3 Preismanagement bei preisabhängiger Qualitätsbeurteilung . . . . . . . . 604

17.3.1 Der Preis als Qualitätsindikator . . . . . . . . . . . . . . . . . . 604

17.3.2 Preispolitische Implikationen der preisabhängigen

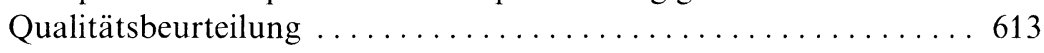

Achtzehntes Kapitel

Preismanagement und Marketing Mix-Interaktion ................ 619

18.1 Preis und Marketing Mix ........................ 619

18.2 Marketing Mix-Interaktion in Modellen ................. 621

18.2.1 Wirkungs- und Entscheidungsinteraktion . . . . . . . . . . 621

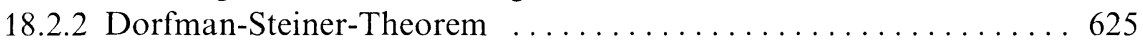

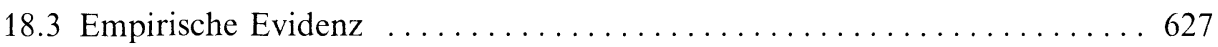

18.4 Ein Modell zur Preis-Werbe-Interaktion . . . . . . . . . . . . . . . . . . 629

18.4.1 Der Zweck der Werbung bezüglich der Preiswirkung . . . . . . . . . 629

18.4.2 Verhalten der Nachfrager $\ldots \ldots \ldots \ldots \ldots \ldots \ldots \ldots \ldots \ldots \ldots \ldots . \ldots \ldots$

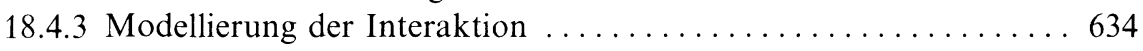

18.4.4 Empirische Befunde ....................... 634

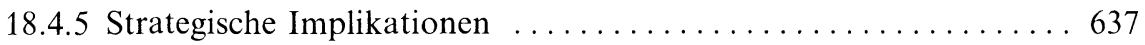

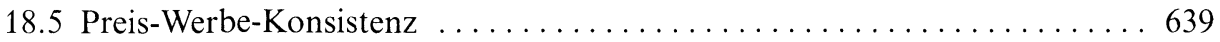


Neunzehntes Kapitel

Umsetzungsprobleme im Preismanagement ...................... 643

19.1 Umsetzung als Herausforderung $\ldots \ldots \ldots \ldots \ldots \ldots \ldots \ldots \ldots \ldots \ldots . \ldots \ldots$

19.2 Zum Proze $ß$ der Preisfindung und Preisentscheidung ............. 643

19.3 Zur Organisation von Preisfindung und Preisentscheidung . . . . . . . . 647

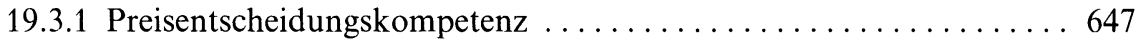

19.3.2 Zur Organisation der Preisfindung .................. 648

19.3.3 Preiskompetenz des Außendienstes . . . . . . . . . . . . . 651

19.4 Zur Umsetzung von Preisen im Markt ................. 655

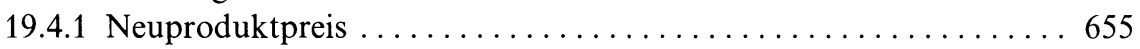

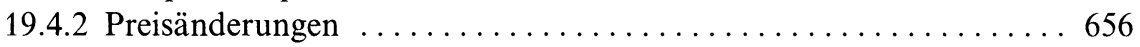

19.4.3 Spezielle Aspekte der Umsetzung . . . . . . . . . . . . . . . 661

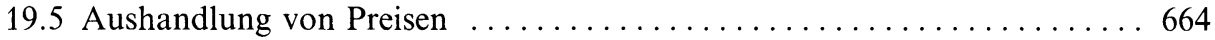

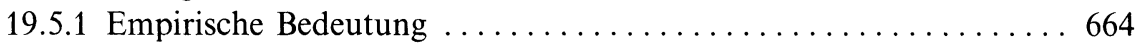

19.5.2 Theorie der Preisaushandlung ....................... 665

19.5.3 Zur Psychologie und Taktik von Preisverhandlungen ......... 670

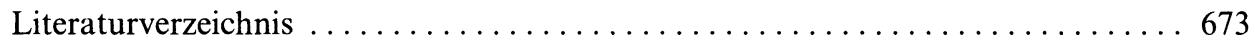

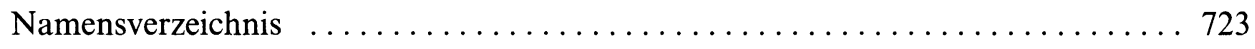

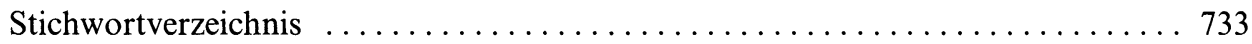

